\title{
Factores de riesgo para infecciones de trasmisión sexual en adolescentes de un colegio de educación secundaria
}

Cifuentes Zúñiga Camila Fernanda ${ }^{1, a}$; Gaete Villegas Guisella Alejandra ${ }^{1, a}$; Sepúlveda Saldias Claudia Mariela ${ }^{1, a}$; Morales Ojeda Ismael Antonio ${ }^{1, \mathrm{~b}}$; Parada Jiménez Darynka Angélica 1,2,a

\section{RESUMEN}

Objetivo: Determinar los factores de riesgo predominantes relacionados con la adquisición de enfermedades de transmisión sexual en adolescentes que cursan secundaria en Coihueco (Chile), durante el segundo semestre del 2018.

Materiales y métodos: Se realizó un estudio de tipo descriptivo y de corte transversal en un grupo de adolescentes de secundaria durante el segundo semestre del 2018. Se utilizó un muestreo simple aleatorio. Los datos fueron procesados por el cálculo porcentual y expresados en tablas. Las variables consideradas fueron sociodemográficas, vínculo familiar, conocimiento y conductas de riesgo para identificar factores predominantes.

Resultados: El rango etario fue 15 a 19 años que cursan 3. ' y 4 . $^{\circ}$ año de educación media. Se evidenció una comprensión de las infecciones de transmisión sexual. Alrededor del 30,00\% de los estudiantes ha iniciado ya sus relaciones sexuales, lo que demuestra la existencia de prácticas de riesgo como edad de inicio sexual cercana a 15 años, uso de drogas, obtención de beneficios a cambio de sexo y no empleo de condón como medio de protección. No se encontraron evidencias de que los estudiantes hayan contraído infecciones de transmisión sexual.

Conclusiones: Ante los factores de riesgo encontrados, es necesario educar respecto de la prevención del contagio de infecciones de transmisión sexual.

Palabras clave: Infecciones sexualmente transmitidas; Adolescentes; Factores de riesgo; Sexualidad (Fuente DeCS: BIREME).

\section{Risk factors for sexually transmitted infections in adolescents from a secondary school}

\section{ABSTRACT}

Objective: To determine the predominant risk factors for sexually transmitted diseases in adolescents from a secondary school in Coihueco (Chile) during the second half of 2018.

Materials and methods: A cross-sectional descriptive study was conducted with a group of secondary school adolescents during the second half of 2018. A simple random sampling was performed. Data were processed using percentage calculation and entered into tables. Family bond, knowledge and risky behaviors were considered as sociodemographic variables in order to identify the predominant factors.

Results: The sample consisted of people aged between 15 and 19 who were in the $3^{\text {rd }}$ and $4^{\text {th }}$ year of secondary education. The students demonstrated they were knowledgeable about sexually transmitted infections. Approximately $30 \%$ had started sexual intercourse, thus showing risky behaviors such as early first sexual intercourse around 15 years of age, drug use, sex in exchange for benefits and lack of condom use as protection. There was no evidence that the students had a sexually transmitted infection.

Conclusions: Considering the risk factors found in the research, it is necessary to educate the adolescents regarding the prevention of sexually transmitted infections.

Keywords: Sexually transmitted diseases; Adolescent; Risk factors; Sexuality (Source: MeSH NLM).

1 Universidad Adventista de Chile. Chillán, Chile.

2 Universidad Pedro de Valdivia. Chillán, Chile.

a Licenciado en Enfermería.

b Doctor en Ciencias Biomédicas.

*Autor corresponsal. 


\section{INTRODUCCIÓN}

Las infecciones de transmisión sexual (ITS) constituyen un grupo heterogéneo de enfermedades que se transmiten de persona a persona a través del contacto sexual (vaginal, anal y oral) y mediante contactos no sexuales, como en el caso de transfusiones de sangre o contaminación con fluidos orgánicos ${ }^{(1)}$. Estas infecciones son causadas por diversos agentes patógenos, como bacterias, virus, protozoos, parásitos, entre otros. Las ITS de mayor prevalencia a escala global son las que se denominan curables, por ejemplo, sífilis, gonorrea, tricomonas y clamidia; y entre las incurables destacan la hepatitis B, el herpes simple, el virus de inmunodeficiencia humana (VIH) y el papiloma humano ${ }^{(2,3)}$. La Organización Mundial de la Salud estima que cada año ocurren, en promedio, 448 millones de casos nuevos de ITS en el mundo, sobre todo en el grupo de adultos entre los 15 y 49 años. Los factores que contribuyen a la propagación y persistencia de estas infecciones son biopsicosociales, económicos, conductuales, político-sanitarios, éticos, religiosos y culturales ${ }^{(4)}$.

En Chile, el Departamento de Epidemiología del Gobierno indica que la tasa de ITS en hombres supera a la de mujeres, y que el grupo etario más afectado es el de 15 a 39 años; además, señala que la sífilis es la ITS con mayor tasa de notificación (5961 casos en 2017), seguida de la infección por VIH (5000 casos nuevos anuales en el mismo año) ${ }^{(5)}$. Existen diversos factores de riesgo que influyen en la infección, como promiscuidad, falta de educación en temas de sexualidad, presión social por experimentar, sentimientos de invulnerabilidad, consumo de alcohol y drogas, no uso continuo de preservativos, entre otros ${ }^{(6,7)}$. Todos ellos son problemas que todavía están presentes en la sociedad chilena a pesar de la implementación de programas de educación sexual en los establecimientos educativos. Si bien existe un avance respecto a ciertos indicadores, como la incorporación de contenidos curriculares, el descenso del número de embarazos adolescentes y el empleo de métodos anticonceptivos, se observa un aumento preocupante de los casos de ITS y una disminución de los métodos que previenen su contagio, hechos que evidencian violencia sexual dirigida hacia los adolescentes, la cual constituye un riesgo para contraer estas enfermedades ${ }^{(8)}$.

En el contexto social chileno, se ha implementado la Ley 21120 , la cual reconoce y da protección al derecho a la identidad de género ${ }^{(9)}$ y regula los procedimientos para rectificar la partida de nacimiento de una persona en lo relativo a su sexo y nombre, cuando esta no se corresponda o no sea congruente con su identidad de género. Estos aspectos han sido incluidos en el colegio estudiado (en la provincia de Ñuble), lugar donde se brinda una educación sin discriminación social, política, religiosa o dogmática de ningún tipo, lo que facilita el acceso a una muestra diversa y heterogénea de estudiantes con diferentes tendencias/identidades sexuales. Frente a los elementos ya mencionados se hace necesario describir los factores de riesgo predominantes que se relacionan con la adquisición de enfermedades de transmisión sexual en adolescentes de un colegio de educación secundaria en la provincia de Ñuble.

\section{MATERIALES Y MÉTODOS}

\section{Diseño y población de estudio}

Estudio de tipo cuantitativo, descriptivo y transversal. La población correspondió a 184 estudiantes de 15-19 años que asisten a un colegio de educación secundaria de la provincia de Ñuble, de cualquier identidad de género. Los estudiantes que no desearon participar y los que no completaron las encuestas fueron excluidos. Antes de la entrega del instrumento se realizó una reunión con la directiva del establecimiento para explicar el proceso de recolección de datos y solicitar autorización para la investigación. Asimismo, quedaron establecidos el uso de un consentimiento informado previo a la encuesta y las normas de privacidad de los datos.

\section{Variables y mediciones}

La encuesta empleada en esta investigación se redactó de acuerdo a dos documentos. El primero fue el Instrumento de trabajo para el estudio de las enfermedades de transmisión sexual y VIH/SIDA en adolescentes ${ }^{(10)}$, validado y sometido al criterio de expertos en La Habana, que registra la información acerca de variables sociodemográficas, conocimientos, actitudes y prácticas sobre el VIH-sida en adolescentes (11). El segundo fue la Encuesta de salud y hábitos sexuales ${ }^{(12)}$, cuya versión original evalúa las características sociodemográficas, estilos de vida, información y experiencia sexual, prueba de VIH, salud sexual y actitudes; y que ha sido aplicado en Estados Unidos y España ${ }^{(13,14)}$.

Las preguntas seleccionadas y adaptadas al contexto local buscaron recolectar la información referente a las características sociodemográficas de la población y la descripción de vínculos familiares, conocimientos y conductas sexuales de riesgo. En el caso de la presente investigación, la estructura de las preguntas se adaptó al contexto estudiantil del colegio de educación secundaria. Los participantes completaron la encuesta solos, para tener privacidad al momento de responder.

\section{Análisis estadístico}

El programa SPSS v. 19 fue empleado para el procesamiento estadístico. Se utilizó la estadística descriptiva para presentar las variables cualitativas en tablas de frecuencia y porcentajes, y las variables cuantitativas se mostraron en medias y desviación estándar.

\section{Consideraciones éticas}

El estudio fue aprobado por el Comité de Bioética de la 
Universidad Adventista de Chile y por las autoridades del colegio en donde se realizó el estudio.

\section{RESULTADOS}

La muestra se compuso de un $44,80 \%$ de personas del sexo masculino. La edad de la muestra es de 16,9 años, y la edad en que comenzaron a tener relaciones sexuales es de 15,3 años. El 1,40\% de los estudiantes encuestados refirió tener hijos, lo que es un porcentaje considerablemente menor de lo esperado. De 184 participantes, solo el $1,70 \%$ es casado; el 39,20\% indica estar en una relación de pareja, y el $60,80 \%$ señala ser soltero.

Sobre el vínculo familiar y satisfacción del adolescente, el
53,60 \% señaló sentirse "siempre" satisfecho con la ayuda que recibe de su familia cuando tiene alguna dificultad. Un $41,50 \%$ refirió "siempre" sentirse satisfecho cuando habla con su familia compartiendo sus problemas. El $48,40 \%$ indicó "siempre" sentirse satisfecho por como su familia lo acepta y lo apoya a emprender nuevas actividades. El 36,20 \% señaló "siempre" sentirse satisfecho con el afecto entregado por su familia. El 55,70 \% refirió "siempre" sentirse satisfecho con el tiempo que comparten junto a su familia. Un $57,10 \%$ de la muestra señaló que "siempre" comparten espacios en la casa. Un 49,70 \% indicó que "siempre" se siente satisfecho con respecto a cómo se distribuye el dinero en el hogar. Un $53,00 \%$ refirió que "siempre" cuenta con un amigo en caso de dificultades (Tabla 1).

Tabla 1. Vínculo familiar y satisfacción del adolescente

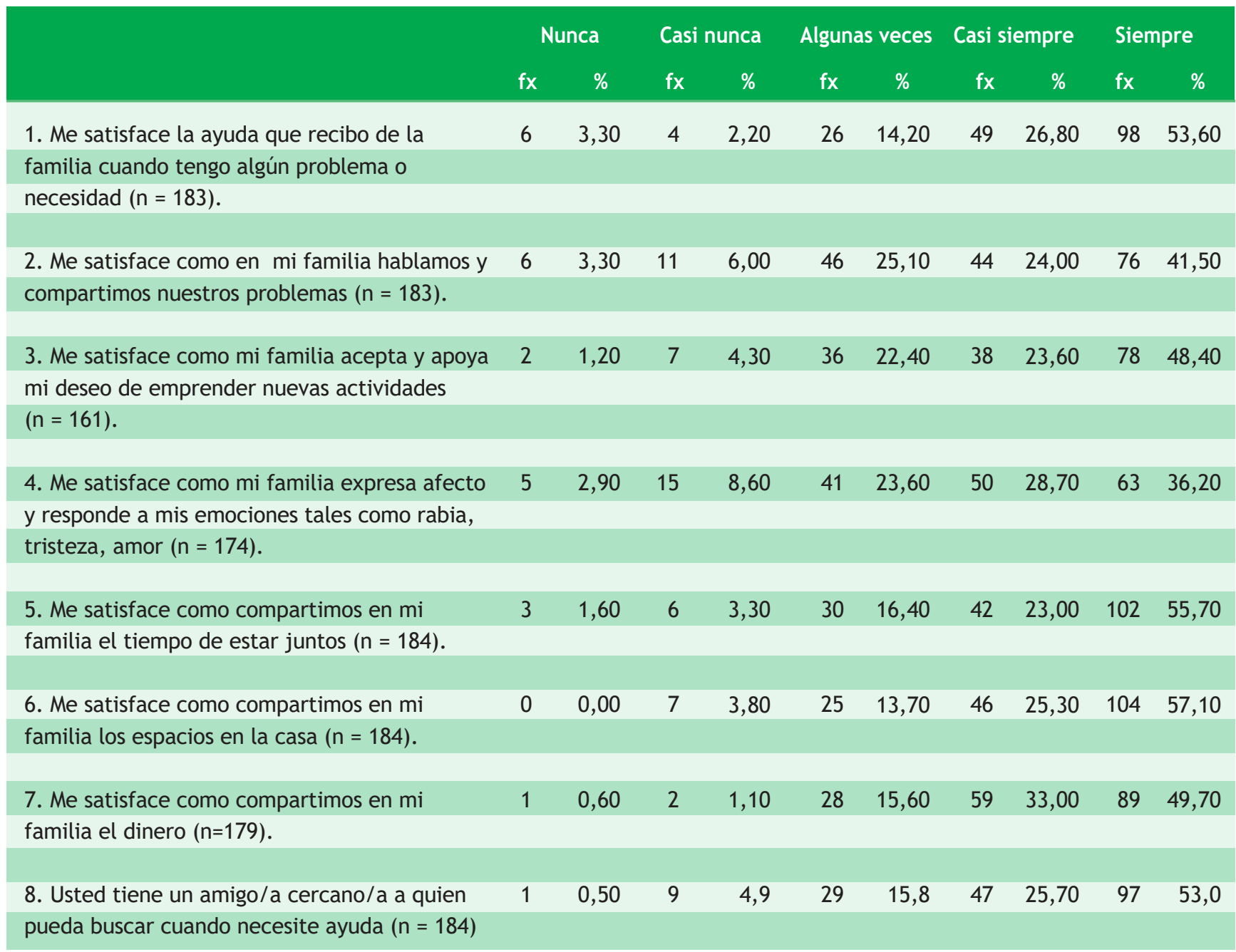

$f x=$ frecuencia 
En la población estudiada, las fuentes de información más importantes son las siguientes: para temas sexuales es la madre $(40,10 \%)$, y para la anticoncepción son los programas de salud ( $45,30 \%$ ) y las madres $(24,90 \%$ ) (Tabla 2$)$.

Tabla 2. Fuente de información relevante en sexualidad

\begin{tabular}{|c|c|c|c|c|}
\hline & \multicolumn{2}{|c|}{$\begin{array}{l}\text { Fuente de información más importante } \\
\text { para usted respecto a temas sexuales }\end{array}$} & \multicolumn{2}{|c|}{$\begin{array}{l}\text { Fuente de información más importante } \\
\text { para usted respecto a anticonceptivos }\end{array}$} \\
\hline & $f x$ & $\%$ & $f x$ & $\%$ \\
\hline Madre & 73 & 40,10 & 45 & 24,90 \\
\hline Pareja & 10 & 5,50 & 7 & 3,90 \\
\hline Profesores & 15 & 8,20 & 12 & 6,60 \\
\hline Sistema de salud & 43 & 23,60 & 82 & 45,30 \\
\hline Nadie & 6 & 3,30 & 7 & 3,90 \\
\hline
\end{tabular}

$f x=f r e c u e n c i a$

De 184 alumnos, el 98,90 \% sabía lo que es una ITS. Por otro lado, el 5,00 \% refiere que un condón se puede utilizar varias veces, y un 4,40 \% no sabe cómo utilizarlo. El 33,10\% de los alumnos utilizan algún método anticonceptivo: el que se emplea con más frecuencia es el anticonceptivo oral (52,80 \%) y el menos usado es el método calendario (2,80 \%).
Con respecto a los métodos anticonceptivos para prevenir las ITS, un 85,20 \% indicó la utilización de condones como mejor método para prevenirlas. Pero es importante destacar que, dentro de la muestra, hubo un porcentaje (14,80\%) que señaló que los anticonceptivos orales protegen de ITS (Tabla 3).

Tabla 3. Conocimiento de anticoncepción para prevenir ITS

\begin{tabular}{|c|c|c|c|c|}
\hline \multirow{2}{*}{$\begin{array}{l}\text { Métodos anticonceptivos que } \\
\text { sirven para prevenir ITS según los } \\
\text { estudiantes del liceo }\end{array}$} & \multicolumn{2}{|c|}{ Sí } & \multicolumn{2}{|c|}{ No } \\
\hline & $f x$ & $\%$ & $f x$ & $\%$ \\
\hline Condón & 163 & 89,10 & 20 & 10,90 \\
\hline Anticonceptivo oral & 27 & 14,80 & 156 & 85,20 \\
\hline Dispositivo intrauterino (DIU) & 9 & 4,90 & 174 & 95,10 \\
\hline Método calendario & 5 & 2,70 & 178 & 97,30 \\
\hline Inyección anticonceptiva & 22 & 12,00 & 161 & 88,00 \\
\hline Lubricante & 1 & 0,50 & 182 & 99,50 \\
\hline Abstinencia & 31 & 16,90 & 152 & 83,10 \\
\hline
\end{tabular}

$f x=$ frecuencia

Con respecto al conocimiento que tienen los estudiantes sobre las enfermedades que pueden contagiarse por contacto sexual, la mayoría indicó conocer el VIH (95,70\%), seguido de la gonorrea $(79,30 \%)$ y de la sífilis $(71,20 \%)$. Por otro lado, los agentes infecciosos menos conocidos por los participantes fueron Gardnerella $(97,80 \%)$, tricomonas $(94,60 \%)$ y clamidias (75,50\%) (Tabla 4).

Tabla 4. Conocimiento acerca de enfermedades e ITS

\begin{tabular}{lrrrrr} 
Enfermedades de transmisión & & \multicolumn{2}{c}{ Sí } & \multicolumn{2}{c}{ No } \\
\cline { 3 - 6 } $\begin{array}{l}\text { sexual, según los estudiantes de } \\
\text { secundaria }\end{array}$ & & $\mathrm{fx}$ & $\%$ & $\mathrm{fx}$ & $\%$ \\
\hline Sífilis $\mathrm{n}=184$ & 131 & 71,20 & 53 & 28,80
\end{tabular}




\begin{tabular}{|c|c|c|c|c|}
\hline \multirow{2}{*}{$\begin{array}{l}\text { Enfermedades de transmisión } \\
\text { sexual, según los estudiantes de } \\
\text { secundaria }\end{array}$} & \multicolumn{2}{|c|}{ Sí } & \multicolumn{2}{|c|}{ No } \\
\hline & $f x$ & $\%$ & $f x$ & $\%$ \\
\hline Tricomonas & 10 & 5,40 & 174 & 94,60 \\
\hline Gardnerella & 4 & 2,20 & 180 & 97,80 \\
\hline Ladillas & 66 & 35,90 & 118 & 64,10 \\
\hline Clamidia & 45 & 24,50 & 139 & 75,50 \\
\hline Gonorrea & 146 & 79,30 & 37 & 20,10 \\
\hline VIH & 176 & 95,70 & 8 & 4,30 \\
\hline Herpes simple & 125 & 67,90 & 59 & 32,10 \\
\hline \multirow{2}{*}{$\begin{array}{l}\text { Drogas que consumen } \\
\text { los estudiantes }\end{array}$} & \multicolumn{2}{|c|}{ Sí } & \multicolumn{2}{|c|}{ No } \\
\hline & $f x$ & $\%$ & $f x$ & $\%$ \\
\hline Marihuana & 8 & 4,40 & 173 & 95,60 \\
\hline Cocaína & 3 & 1,70 & 178 & 98,30 \\
\hline Pasta base & 3 & 1,70 & 178 & 98,30 \\
\hline Benzodiacepina (clonazepam, diazepam) & 3 & 1,70 & 178 & 98,30 \\
\hline Otras & 6 & 3,30 & 174 & 96,70 \\
\hline Ninguna & & & 169 & 100,0 \\
\hline
\end{tabular}

\section{$f x=f r e c u e n c i a$}

El 43,00\% de encuestados refiere no usar condón al momento de tener relaciones sexuales; igualmente, el $93,90 \%$ solo tiene relaciones con personas de sexo opuesto, y una minoría mantiene relaciones bisexuales u homosexuales. Es preocupante que un porcentaje de participantes $(2,30 \%)$ refiera haber accedido a tener encuentros sexuales a cambio de dinero o regalos (beneficios). Sobre las conductas de riesgo frente a las relaciones sexuales, los encuestados señalaron que no emplearon el condón en su última relación sexual porque esta fue casual o inesperada $(19,10 \%)$, porque no les gusta usar el preservativo $(17,60 \%)$ o por otros motivos (14,70\%) (Tabla 5).

Tabla 5. Conductas de riesgo

\begin{tabular}{|lrr|}
\hline $\begin{array}{l}\text { Razón por la que no utilizó condón } \\
\text { durante su última relación sexual }\end{array}$ & fx & \multicolumn{1}{c|}{$\%$} \\
\hline Estaba bajo el efecto del alcohol & 4 & 5,90 \\
\hline Fue una relación casual o inesperada & 13 & 19,10 \\
\hline No le gusta usar condón & 12 & 17,60 \\
\hline No tenía condón en ese momento & 6 & 8,80 \\
\hline Por confianza de su pareja & 23 & 33,80 \\
\hline Otro & 10 & 14,70 \\
\hline Total & 68 & 100,00 \\
\hline fx=frecuencia & & \\
\hline
\end{tabular}

El 3,60\% de los menores refiere que alguna vez en su vida alguien los obligó a tener relaciones sin su consentimiento. Respecto al número de parejas, el 3,60\% ha tenido más de
4; un 45,3\%, solo una pareja, y 38,00 \% nunca ha tenido pareja sexual. 


\section{DISCUSIÓN}

Los estudiantes indican que tienen el apoyo de su entorno familiar ante problemas o dificultades, tema que es de vital importancia en lo relacionado con la creación de un ambiente de amor y confianza que facilite el diálogo enfocado a la enseñanza de conocimientos y prácticas sobre sexualidad, lo que mejora el autoconcepto ${ }^{(15,16)}$. En el hogar, los recursos económicos están disponibles de manera equitativa para satisfacción en los encuestados. Este aspecto se toma como un factor protector, puesto que para los jóvenes el recurso económico y la educación oportuna permiten la accesibilidad a métodos de protección y autonomía en temas de sexualidad ${ }^{(17)}$.

Las amistades pueden considerarse como una red de apoyo para buscar ayuda (siempre o casi siempre, en el 75,00\% de los encuestados). Este es un aspecto importante, ya que en otras investigaciones las amistades pueden considerarse como un factor de riesgo porque fomentan el consumo de drogas o la promiscuidad, lo que ocasiona resultados negativos, como el abandono de los estudios, contagio de ITS o embarazo no deseado ${ }^{(15,18)}$.

En el área de conocimiento, casi la totalidad de los encuestados reconoce saber qué es una ITS y cuáles son las más comunes. Es probable que este hecho se asocie a las campañas de publicidad y salud pública realizadas en Chile ${ }^{(19)}$. Asimismo, los estudiantes mostraban confusión para reconocer otras ITS menos comunes o aquellas menos difundidas por los programas educativos, lo que se asociaría al mayor énfasis que se da al VIH y a la sífilis. Esto se considera un factor de riesgo, ya que puede existir posibilidad de contagio a causa de la falta de información.

Según el análisis de conocimientos sobre anticoncepción, los anticonceptivos orales, la abstinencia y la inyección se reconocen como los principales métodos. En algunos casos, se confunde la prevención de embarazos con la prevención de infecciones de transmisión sexual, lo que indicaría la necesidad de entregar información más precisa en las instituciones educativas y de atención primaria, tal como se expone en el Plan Nacional de Prevención y Control del $\mathrm{VIH} / \mathrm{SIDA}$ e ITS $2018-2019{ }^{(19)}$. Las fuentes primordiales de información sobre temas sexuales y de anticoncepción serían la figura materna y el sistema público de salud; este último sería el canal más confiable de la información que es entregada al alumnado dentro del programa educativo.

Sobre las conductas de riesgo, encontramos que las drogas ilícitas se utilizan con poca frecuencia y que, de ellas, la marihuana es la sustancia más consumida. Esta droga se asocia a las prácticas de riesgo en mujeres, particularmente a las relaciones sexuales sin condón $(\mathrm{OR}=2,96, \mathrm{IC} 95 \%: 1,52-5,75)^{(20)}$. En el ámbito de la relación sexual, del total de los encuestados, más del 90,00\% ha practicado el coito con personas del sexo opuesto, a pesar de ser menores de 18 años. El 43,00 \% refiere no usar condón durante la fase de penetración, lo que se considera como un factor importante para contraer una infección de transmisión sexual ${ }^{(20)}$. Las principales justificaciones para esta conducta son las siguientes: las relaciones casuales o inesperadas, tener una sola pareja y el desagrado que ocasiona el uso del preservativo. Se plantea que los adolescentes deberían contar con métodos de prevención contra las ITS y el embarazo desde el inicio de las relaciones sexuales ${ }^{(15)}$.

Por otro lado, se considera como un factor protector que el $45,30 \%$ de los participantes haya tenido solo una pareja sexual. Sin embargo, existe el riesgo de que luego esta población tenga, a edades muy tempranas, amigos o compañeros sexuales ocasionales, lo que puede traer graves consecuencias respecto a las ITS, sobre todo en las mujeres ${ }^{(18)}$. En España, un estudio similar realizado con adolescentes de 14 años mostró resultados similares, el $17,00 \%$ de estudiantes había tenido relaciones con penetración y mostraron los siguientes factores de riesgo: peor relación con los padres, divorcio de los padres, fumar, beber alcohol/emborracharse, consumir drogas, peor salud actual, peor alimentación, peor memoria, erotofilia y ser agnóstico/ateo (21).

En nuestro estudio, el factor de riesgo primordial es la dificultad para identificar las infecciones de transmisión sexual menos conocidas. Existe también una confusión entre los métodos de anticoncepción y los mecanismos para prevenir las enfermedades de trasmisión sexual. Por ello, es alarmante la baja incidencia del uso voluntario de métodos de barrera: la población toma estos riesgos con base en argumentos subjetivos, como confianza en la pareja, relaciones casuales, entre otros ${ }^{(21)}$.

Por último, aunque el porcentaje obtenido es bajo (2,30 \%), destaca el tema de acceder a tener relaciones sexuales a cambio de dinero, regalos o beneficios, lo que indicaría un posible inicio en la prostitución. Respecto a las relaciones sexuales sin consentimiento, el 3,60\% confirmó que en algún momento de su vida se vieron expuestos a este tipo de situación, lo cual incrementa el riesgo de ITS y es atribuible al consumo de drogas y a la promiscuidad sexual (21).

Respecto a los factores de riesgo relacionados con la adquisición de enfermedades de transmisión sexual en adolescentes, concluimos que se observan distintos factores que constituyen un riesgo para el desarrollo saludable de los individuos, a los que la influencia del sistema educativo aminora parcialmente. Pero es necesario establecer intervenciones de atención primaria que permitan modificar los contextos sociales de desarrollo de los adolescentes, facilitar el acceso a la información y a métodos anticonceptivos y de prevención de ITS, con 
intervención educativa familiar orientada hacia la práctica de la sexualidad responsable.

Contribuciones de los autores: Ismael Morales Ojeda realizó el procesamiento estadístico y la revisión final del manuscrito. Darynka Parada Jiménez y Camila Fernanda Cifuentes Zúñiga seleccionaron la muestra del estudio y elaboraron el manuscrito. Gaete Villegas Guisella Alejandra y Sepúlveda Saldias Claudia Mariela, realizaron la aplicación de instrumentos y la elaboración del manuscrito.

Fuentes de financiamiento: El artículo ha sido financiado por los autores.

Conflictos de interés: Los autores declaran no tener conflictos de interés.

\section{REFERENCIAS BIBLIOGRÁFICAS}

1. Martins DC, Pesce GB, Silva GM, Fernandes CA. Sexual behavior and sexually transmitted diseases among the female partners of inmates. Rev Latino-Am Enfermagem. 2018; 26: e3043.

2. Chacón-Quesada T, Corrales-González D, Garbanzo-Núñez D, Gutiérrez-Yglesias JA, Hernández-Sandí Alejandro, Lobo-Araya Adriana et al. ITS Y SIDA en adolescentes: descripción, prevención y marco legal. Med Leg Costa Rica. 2009; 26(2): 79-98.

3. Pérez-Morente MA, Campos-Escudero A, Sánchez-Ocón MT, HuesoMontoro C. Características sociodemográficas, indicadores de riesgo y atención sanitaria en relación a infecciones de transmisión sexual en población inmigrante de Granada. Rev Esp Salud Publica. 2019; 93: e201912102.

4. Blandón-Buelvas M, Palacios-Moya L, Berbesí-Fernández D. Infección activa por sífilis en habitantes de calle y factores asociados. Rev Esp Salud Publica. 2019; 21(3): e461039.

5. Cáceres-Burton K. Informe: Situación epidemiológica de las infecciones de transmisión sexual en Chile, 2017. Rev Chil Infectol. 2019; 36(2): 221-33.

6. Heredia LPD, Ramirez EGL, Pereira CF, Vargas D. Efeito das variáveis sociodemográficas e de vulnerabilidade no padrão do uso de álcool em mulheres universitárias. Texto contexto - Enferm. 2017; 26(3): e6860015.

7. Spindola T, Araújo A, Brochado E, Marinho D, Martins E, Pereira T. Prácticas sexuales y comportamiento de jóvenes universitarios frente a la prevención de infecciones de transmisión sexual. Enferm Glob. 2020; 19(58): 109-40.

8. Castro-Sandoval G, Carrasco-Portiño M, Solar-Bustos F, CatrienCarrillo M, Garcés-González C, Marticorena-Guajardo C. Impacto de las políticas de educación sexual en la salud sexual y reproductiva adolescente en el sur de chile, período 2010-2017. Rev Chil Obstet Ginecol. 2019; 84(1): 28-40.

9. Ministerio de Justicia y Derechos Humanos de Chile. Ley 21120. Reconoce y da protección al derecho a la identidad de género [Internet]. Santiago: Biblioteca del Congreso Nacional de Chile; 2018. Disponible en: https://www.bcn.cl/leychile/ navegar?idNorma $=1126480$

10. Cortés A, García R, Fullerat R, Fuentes J. Instrumento de trabajo para el estudio de las enfermedades de transmisión sexual y $\mathrm{VIH} /$ SIDA en adolescentes. Rev Cubana Med Trop. 2000; 52(1): 48-54.

11. Contreras-Britto JB, Trout-Guardiola G. Conocimientos, actitudes y prácticas sobre $\mathrm{VIH}$-sida en adolescentes de $9^{\circ}, 10^{\circ}$ y $11^{\circ}$ grado de un colegio público del Distrito de Santa Marta-Colombia. Duazary. 2018; 15(3).
12. Instituto Nacional de Estadística de España. Encuesta de salud y hábitos sexuales, 2003. Resultados: Microdatos [Internet]. Madrid; 2004. Disponible en: https://www.ine.es/dyngs/INEbase/es/ operacion. htm?c=Estadistica_C\&cid=1254736176785\&menu=resulta dos\&idp $=1254735573175 \#$ !tabs-1254736194731

13. Instituto Nacional de Estadística de España. Encuesta de salud y hábitos sexuales, 2003. Metodología [Internet]. Madrid; 2004 Disponible en: https://www.ine.es/dyngs/INEbase/es/operacion. $\mathrm{htm}$ ?c=Estadistica_C\&cid=1254736176785\&menu=metodologia\& $i$ $\mathrm{dp}=1254735573175$

14. Navarro B, Ros L, Latorre JM, Escribano JC, López V, Romero M. Hábitos, preferencias y satisfacción sexual en estudiantes universitarios. Rev Clín Med Fam. 2010; 3(3): 150-7.

15. Mendoza LA, Claros DI, Peñaranda CB. Actividad sexual temprana y embarazo en la adolescencia: estado del arte. Rev Chil Obstet Ginecol. 2016; 81(3): 243-53.

16. Reynoso OM, Caldera JF, de la Torre V, Martínez A, Macías GA. Autoconcepto y apoyo social en estudiantes de bachillerato. Un estudio predictivo. RPCC-UACJS. 2018; 9(1): 100-19.

17. Da Fonte V, Pinheiro C, Barcelos N, Costa C, Ribeiro F, Spindola T. Factores asociados con el uso del preservativo entre hombres jóvenes que tienen sexo con hombres. Enferm Glob. 2017; 16(46): 50-93.

18. García H, Soriano E. "Amigos con beneficios": salud sexual y estilos de apego de hombres y mujeres. Saude Soc. 2016; 25(4): 1136-47.

19. Ministerio de Salud, Gobierno de Chile. Plan Nacional de Prevención y Control del VIH/SIDA e ITS 2018-2019 [Internet]. Santiago de Chile; 2019. Disponible en: https://diprece.minsal.cl/wp-content/ uploads/2019/06/2019.06.12_PLAN-NACIONAL-VIH-SIDA-E-ITS.pdf

20. Moure-Rodríguez L, Doallo S, Juan-Salvadores P, Corral M, Cadaveira F, Caamaño-Isorna F. Consumo intensivo de alcohol y cannabis, y prácticas sexuales de riesgo en estudiantes universitarios. Gac Sanit. 2016; 30(6): 438-43.

21. Royuela P, Rodríguez L, Marugán J, Carbajosa V. Factores de riesgo de la precocidad sexual en adolescentes. Rev Pediatr Aten Primaria. 2016; 17(66): 127-36.

\section{Correspondencia:}

Ismael Antonio Morales Ojeda

Dirección: Las Mariposas, km 12, Chillán, Región de Ñuble, Chile.

Teléfono: +56 995492358

Correo electrónico: ismaelmoralesojeda@gmail.com

Recibido: 25 de noviembre de 2019

Evaluado: 23 de octubre de 2020 Aprobado: 07 de diciembre de 2020

(c) La revista. Publicado por Universidad de San Martín de Porres, Perú (c) ву Licencia de Creative Commons Artículo en acceso abierto bajo términos de Licencia Creative Commons Atribución 4.0 Internacional. (http://creativecommons.org/licenses/by/4.0/)

\section{ORCID iDs}

Cifuentes Zúñiga, Camila Fernanda @ https: / / orcid.org/0000-0001-8821-1686 Gaete Villegas, Guisella Alejandra 은 htps://orcid.org/0000-0002-3966-124X Sepúlveda Saldias, Claudia Mariela 근 https://orcid.org/0000-0003-3997-6230 Morales Ojeda, Ismael Antonio (1) https: / / orcid.org/0000-0002-1752-7023 Parada Jiménez, Darynka Angélica ㄴo https: / / orcid.org/0000-0002-7497-8330 